rechtlich übertragen. Die Vertragsstaaten des Internationalen Übereinkommens von 1979 über den Such- und Rettungsdienst auf See sind verpflichtet, Such- und Rettungsdienste einzurichten und dabei international zu kooperieren.59

Ginge es den EU-Mitgliedstaaten primär um die Rettung von Menschenleben, müsste sich dies auch im institutionellen Gefüge niederschlagen: Nicht der Ausbau von Frontex, sondern der Seenotrettungsdienste müsste vorangetrieben werden. Solange das nicht geschieht, muss sich die EU mangelnde Glaubwürdigkeit vorwerfen lassen.

\title{
Dario Azzellini
}

\section{Die neuen Söldner}

Bezahlte Erbringer von militärischen Dienstleistungen, Söldner genannt, sind in der Kriegsgeschichte nicht unbekannt. Mit der Erfindung der Privaten Militärunternehmen, der Private Military Contractors (PMC) und Private Security Contractors (PSC) Anfang der I990er Jahre, wurde das Geschäft allerdings in den vermeintlich »sauberen« Wirtschaftsbereich überführt. Somit können die Unternehmen offen rekrutieren und ihre Dienstleistungen auf dem Weltmarkt anbieten.

Die Industrie der neuen Söldner hat sich in den vergangenen 20 Jahren weltweit entwickelt, und PMC und PSC aus den USA, Großbritannien, Israel, Frankreich, Deutschland, Australien, Russland, Rumänien, Bulgarien und anderen Länder bieten Dienstleistungen jeder Art an. Hoch professionelle Unternehmen aus den USA und Großbritannien stellen jedoch mehr als 70 Prozent der Militärdienstleistungen rund um den Globus zur Verfügung. ${ }^{2}$ Sie kommen vor allem im Irak und Afghanistan, aber auch in Kolumbien, Somalia, Sudan und anderen Ländern zum Einsatz. ${ }^{2}$

Im Irak sind sogar mehr Angehörige von PMC und PSC im Einsatz als Soldaten aller internationalen Besatzungsmächte zusammen. Die Los Angeles Times berichtete am 4. Juli 2007 mit Berufung auf Informationen der US Army, im Irak seien I 80.000 PMC- und PSC-Angehörige unter Vertrag. ${ }^{3}$ Ihnen obliegt es, Patrouille zu laufen, Gebäude und Infrastruktur zu bewachen, Personenschutz zu stellen und Militärgefängnisse zu führen. Schon bei der Invasion wurden viele der hoch entwickelten Waffensysteme von Spezialisten bedient, wie etwa die unbemannten Predator-Drohnen, die Global Hawks oder auch die B-2 StealthBomber.4 Aber auch Privatunternehmen greifen auf die Dienste von PMF zurück. Daher wird die coalition of the willing häufig auch als coalition of the billing (Koalition der Rechnungssteller) bezeichnet.

59 Vgl. insbesondere Kapitel 2 und 3 des genannten Übereinkommens.

I www.sourcewatch.org.

2 United Nations, Report of the Working Group on the use of mercenaries as a means of violating human rights and impeding the exercise of the right of peoples to self-determination, 9.I.2008.

3 Christian Miller, Private contractors outnumber US troops in Iraq, Los Angeles Times vom 4.7.2007.

4 Traynor, Ian, The privatisation of war, The Guardian vom I0.I 2.2003. 
$\mathrm{Zu}$ den Großverdienern im Irak gehört das US-Unternehmen Halliburton, in dessen Vorstand bis vor einigen Jahren auch US-Vizepräsident Dick Cheney saß. Das Auftragsvolumen für die verschiedenen Dienstleistungen Halliburtons im Rahmen des Irak-Krieges betrug bis 2007 I,7 Milliarden US-Dollar, 7 Io Millionen Dollar davon für die Instandsetzung und den Betrieb der Ölproduktion. In der Türkei, Jordanien und Kuwait kümmert sich Halliburton um Unterkünfte und Logistik der US Army und bekam dafür gleich zu Beginn des Krieges 390 Millionen US-Dollar. Das bis vor kurzem noch Halliburton-Tochterunternehmen Kellogg, Brown \& Root erhielt allein in den ersten Kriegsjahren 170 Millionen Dollar für Wiederaufbaumaßnahmen, 28 Millionen Dollar für den Bau von Kriegsgefangenenlagern, 269 Millionen Dollar für den Bau von Unterkünften und den Transport von US-Army-Angehörigen und weitere 40 Millionen Dollar für die Bereitstellung der Logistik für die Suche nach den vermeintlichen Massenvernichtungswaffen.'

Und sogar viele der Militärgefängnisse im Irak werden von Privatfirmen betrieben. Dabei werden auch Verhörspezialisten und Dolmetscher über PMC angestellt. So waren Mitarbeiter der PMC Caci und Titan in die Foltervorfälle im Abu-Ghraib-Gefängnis verstrickt. Darin liegt auch die Erklärung, warum bezüglich der Foltervorfälle in Abu-Ghraib nur zehn Soldaten vor Gericht kamen: Ein Großteil der Verhöre und auch der Gefängnissicherheit oblag Mitarbeitern der privaten Militärunternehmen Caci und Titan. Diese unterliegen, da sie keine Angehörigen der US Army sind, nicht der Militärgerichtsbarkeit.

Ende Dezember 2007 haben mit Unterstützung des Center for Constitutional Rights (CCR) 256 ehemalige Abu-Ghraib-Gefangene Klage gegen Caci International wegen Folter eingereicht. Sie sagen aus, 2003 und 2004 u.a. wiederholt geschlagen, sexuell missbraucht, mit Vergewaltigung bedroht, nackt in Zellen eingesperrt, mit Elektroschocks gefoltert und mit Hunden attackiert worden zu sein. Die Klage benennt konkret zwei Söldner, welche bereits wegen Folter verurteilte US Army Angehörige angeleitet und befehligt hätten. ${ }^{6}$

\section{Gewalttätige Mente?}

Folter, gewalttätige Übergriffe und selbst Morde durch PMC-Mitarbeiter sind im Irak an der Tagesordnung. Mitte November 2007 erschoss ein DynCorpAngestellter einen Taxifahrer, »einfach so und dann sind sie weiter gefahren«, erklärte General Abdul Karim Khalaf, Sprecher des irakischen Innenministeriums. ${ }^{7}$ Im Internet kursierten auch schon mit Mobiltelefonen aufgenommene Videos, auf denen feixende Söldner zu sehen waren, die als Zeitvertreib auf fahrende Autos schießen. Und im August 2007 erschossen Söldner der australischen Unity Ressources Group (URG), die im Irak NGO’s eskortiert, zwei Frauen. ${ }^{8}$ Ein Untersuchungsbericht einer Kommission des US-Repräsentantenhauses zu dem US-Unternehmen Blackwater zeichnete im September 2007 ein erschrekkendes Bild. Es ist von »häufiger und umfassender Gewalt« der Blackwater-Mitarbeiter die Rede. Diese seien seit 2005 in mindestens i95 Feuergefechte mit mindestens 16 irakischen Opfern verwickelt gewesen. Dabei haben die vertrag-

\footnotetext{
5 Dago Langhans, Kriegsprofite, Junge Welt vom 4.9.2004.

6 District Court Columbia, Case No. 05-cv-I I65 (JR), Klageerweiterung vom I 7.I 2.2007, der Schriftsatz ist abrufbar unter (Mai 2008): http://ccrjustice.org/files/Saleh_FourthAmendedComplaint_I2_07.pdf

7 TeleSUR vom I 4.I I.2007.

8 TeleSUR vom I 4.I I.2007.
} 
lich zum »Schutz « eingesetzten Blackwater-Mitarbeiter in I 63 Fällen zuerst das Feuer eröffnet. ${ }^{9}$

Der aufsehenerregendste Zwischenfall hatte sich zum Zeitpunkt des Berichtes noch nicht ereignet. Am I6. September 2007 erschossen Blackwater-Söldner am Nisoor-Platz in Bagdad I 7 Zivilisten und verletzten weitere 27 zum Teil schwer. Die Söldner behaupteten, in Notwehr geschossen zu haben. Das verneinten aber nicht nur die irakischen Behörden, sondern auch eine Untersuchung des FBI, die keine Notwehrsituation erkennen konnte und die Erschießung von mindestens I4 der I7 Getöteten als nachweislich völlig ungerechtfertigt bezeichnete. ${ }^{\text {I }}$ Blackwater hatte einen Konvoi begleitet und, nachdem in größerer Entfernung eine Detonation erfolgte, einfach mit Maschinengewehren und Granatwerfern das Feuer auf Fahrzeuge und Zivilisten eröffnet. Anschließend schossen sich die Söldner, I $50 \mathrm{~m}$ vom ersten Tatort entfernt, den Weg an einer verkehrsreichen Kreuzung frei. ${ }^{\text {I }}$ Die irakische Regierung setzte daraufhin die Lizenz Blackwaters aus, doch schon wenige Wochen später zeigte sich, wer im Irak das Sagen hat, und Blackwater operierte wieder wie zuvor. Die US-Regierung sicherte den Blackwater-Söldnern zudem die gleiche Immunität vor US-Zivilklagen zu, wie sie auch US-Militärangehörige genießen. Dabei war die gesonderte Zusicherung gar nicht notwendig. Donald Rumsfeld klassifizierte die PMC bereits im Februar 2006 als Teil des US-Militärapparates, womit sie nicht der Ziviljustiz unterliegen. Und einen Tag vor Ende seines Mandats, am 27. Juni 2004, hatte der US-Irak-Gouverneur Paul Bremer ein Dekret unterzeichnet, das den PMC und PSC Immunität vor der irakischen Justiz verleiht. ${ }^{22}$ Doch zugleich wurden sie nicht der Militärgerichtsbarkeit unterstellt, womit sie völlig straflos handeln können. ${ }^{23}$

Blackwater, I 997 von dem rechtsradikalen Millionär Erik Prince gegründet, hat nicht viel zu befürchten. Seine Privatarmee, die über eine eigene Militärbasis, 20 Flugzeuge und über 20.000 vermittelbare Kriegsspezialisten verfügt, hat allein 2006 für den Irak von der US Regierung Aufträge in Höhe von rund 500 Millionen US Dollar erhalten, 2006 waren es noch mal I, 2 Milliarden Dollar und in den

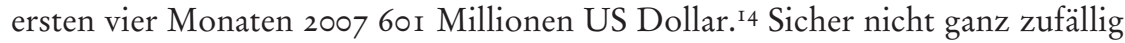
sitzt im BlackwaterDirektorium auch Cofer Back, oberster Verantwortlicher des US-Antiterrorkampfes von 2002 bis 2004. Als Direktor für Operationen und allgemeiner Berater findet sich bei Blackwater auch Joseph Schmitz, ehemaliger Generalinspekteur des Pentagon. ${ }^{\text {Is }}$ Nicht wenige der Söldner haben durchaus zweifelhafte Biographien. So stammt ein großes Kontingent aus Südafrika, meist ehemalige Angehörige von Sondereinheiten des ehemaligen Apartheidregimes wie etwa »Bataillon 32«, Vlakplaas und der Eliteeinheit Koevoet, die in Namibia Terroroperationen gegen Regimegegner durchführten und wegen Menschenrechtsverbrechen aus der Armee entlassen wurden. ${ }^{16}$

Um die Firmen selbst und ihre Chefs sieht es auch nicht besser aus. So etwa im Fall der südafrikanischen Meteoric Tactical Solutions (Hauptbüro in Pretoria, Südafrika), die mit einem Vertrag über 270.000 britische Pfund von der briti-

9 Committee on Oversight and Government Reform, Memorandum vom I.10.2007, abrufbar unter (Mai 2008): http://oversight.house.gov/documents/2007100I I 2 I609.pdf.

Io TeleSUR vom I 4.I I.2007.

I I Spiegel online vom 5.10.2007.

I 2 Coalition Provisional Authority Order Number I7 (Revised) vom 27.6.2004.

I 3 Ùltimas Noticias vom I4.10.2007

I4 Washington Post vom 23.I 2.2007.

Is Spiegel online vom 2.10.2007; TeleSUR vom I 4.I0.2007, 30.10.2007 und vom 8.I I.2007.

I6 Beispiele werden genannt in: La Jornada vom 8.4.2004 und die tageszeitung vom 3.6.2004. 
schen Entwicklungshilfebehörde DFID (Department for International Development) engagiert wurde und Leibwachen und Fahrer für den kleinen Personalstab der DFID im Irak stellt. Zwei der Firmeneigner wurden im März 2004 gemeinsam mit dem zwielichtigen britischen Ex-SAS-Kämpfer Simon Mann verhaftet. Am 7. März 2004 beschlagnahmten die Sicherheitskräfte des Flughafens von Harare in Simbabwe eine mit Waffen beladene Boeing 727 und verhafteten 65 Söldner verschiedener Nationalität. Die Boeing sollte nach ÄquatorialGuinea weiterfliegen, um sich mit einem anderen Söldnerkommando zu treffen. Die Gruppe war beauftragt, einen Putsch zum Sturz des Präsidenten durchzuführen. Simon Mann wiederum hatte zuvor eine Führungsposition in dem mittlerweile aufgelösten südafrikanischen Söldnerunternehmen Executive Outcome. Aus diesem ging Sandline hervor, das Simon Mann gemeinsam mit Anthony Buckingham und dem Ex-SAS-Mitglied Tim Spicer gründete. 1998 wurde Sandline von der britischen Regierung unter Vertrag genommen, um in Sierra Leone zu intervenieren und das UNO-Waffenembargo zu umgehen. Eine maßgebliche Rolle spielte dabei Tim Spicer. Ein Jahr zuvor waren Spicer und Sandline in schwere Bedrängnis gekommen, als sie in einem undurchsichtigen Deal engagiert wurden, um in Papua-Neuguinea illegal die Rebellenarmee von Bouganville zu bekämpfen, die sich gegen den zerstörerischen Kupferabbau zur Wehr setzt. Dennoch erhielt Spicer mit seinem Mitte 2003 gegründeten neuen Unternehmen Aegis Defense Services von der US Army einen Auftrag mit einem Volumen von 293 Millionen US Dollar. Aegis Defense Services stellt für verschiedene, am »Wiederaufbau « des Iraks beteiligte Firmen, zusammen mit dem Personal des Project Management Office (PMO), das die verschiedenen Projekte im Irak leitet, den Schutz und koordiniert die Arbeit der gesamten PMCs in diesem Bereich.

In den vergangenen fünf Jahren haben die PMC Lateinamerika als Rekrutierungsfeld entdeckt. Kriegserfahrene Soldaten gibt es dort nach Befriedung der meisten bewaffneten Konflikte zu genüge. So verlegte zum Beispiel Triple Canopy aus Lincoln, Illinois, seine Rekrutierungstätigkeit von den Philippinen nach El Salvador und Peru. Die Militärgeschichte des zentralamerikanischen Landes sei dafür, Firmensprecher Joe Mayo zufolge, durchaus ein Grund gewesen. Das auf Personenschutz für zivile Mitarbeiter der US-Behörden im Irak spezialisierte Unternehmen war auf der Suche nach Soldaten mit Spezialkräfteausbildung. In El Salvador, während des Bürgerkrieges zwischen I98 I und I993 ein Exerzierfeld für Aufstandsbekämpfung, waren erprobte, von der US-Armee ausgebildete Todesschwadrone nicht schwer zu finden. Und so ist es auch ein ehemaliger US-Militär und früherer Ausbilder der Spezialeinheiten der Armee El Salvadors, der die Rekrutierungsgespräche eingefädelt hat. Der Mann, Teilhaber eines Sicherheitsunternehmens, der gegenüber der Presse anonym bleiben möchte, berichtet, dass 100 Rekruten bereits am I7. September 2004 in Richtung Irak aufgebrochen seien; weitere Gruppen sind seitdem gefolgt. In El Salvador verdienen Leibwächter 350 US-Dollar im Monat - Triple Canopy bietet ihnen I 700 US Dollar. In Peru rekrutiert Triple Canopy direkt über eine von US Personal geführte Niederlassung in Lima. Seit 2005 wurden über 1.000 peruanische Ex-Soldaten aus den berüchtigten antisubversiven Einheiten für Einsätze im Irak rekrutiert.

Gemeinsam mit Blackwater hat Triple Canopy, gemäß Angaben des Senators Alejandro Navarro der regierenden Sozialistischen Partei (PS), seit 2003 mittels Subunternehmern wie die Red Táctica Consulting Group, über I.200 Ex-Militärs aus Chile für Einsätze im Irak rekrutiert. ${ }^{17}$ Viele von ihnen waren wegen 
ihrer Verstrickung in Menschenrechtsverbrechen aus dem Dienst entlassen worden. Über die uruguayische Firma Neskowin, die von José Miguel Pizarro, einem früheren Offizier der Pinochetarmee, geleitet wird, sind sie nun im Irak im Einsatz. Wie Pizarro der chilenischen Zeitung »La Tercera « erklärte, wirbt er auch Ex-Militärs aus Argentinien an. Nach einem Bericht der guatemaltekischen Zeitung Prensa Libre ${ }^{18}$ werden auch in Guatemala massiv ehemalige Angehörige der Armee-Eliteeinheit Kaibiles für Einsätze im Irak rekrutiert. Die Kaibiles waren im Krieg in Guatemala dafür bekannt, ihre Opfer mit Macheten zu verstümmeln.

\section{Privatisierung aus Gründen der Kostenersparnis?}

Die Privatisierung militärischer Dienstleistungen folgt - gemäß des offiziellen Diskurses - der Kostenrationalisierung. Laut neoliberaler Parameter soll der Markt Leistungen grundsätzlich zu besseren Preisen anbieten können als der Staat. Ob im Outsourcing von Militäraufgaben tatsächlich der viel beschworene ökonomische Vorteil liegt, ist allerdings ausgesprochen fraglich. Die zum Einsatz kommenden Waffen werden entweder vom Auftraggeber angeboten oder demselben in Rechnung gestellt. Die Auswahl der Rekruten und Ausbildung der Militärs bleiben Aufgabe der nationalen Armeen und werden nicht von PMC übernommen. So kostet zum Beispiel die i 8monatige Ausbildung eines US-amerikanischen Green Beret rund 250000 US-Dollar. Wenn dieser Soldat anschließend zu einer PMC wechselt, ist mindestens das Dreifache des vorherigen Lohnes fällig.

Zugleich werden die vermeintlichen finanziellen Einsparungen auch durch andere Geschehnisse in Zweifel gestellt. PMC sind als Unternehmen darauf ausgerichtet, den größtmöglichen Profit bei geringstem Einsatz zu erwirtschaften. Damit gerät die Frage des Ausmaßes an »gelieferter« Sicherheit zwangsläufig zu einer Kosten-Nutzen-Rechnung, und damit wird es von einem Allgemeingut zu einer Frage der Finanzstärke. Sicherheit ist damit nicht mehr ein Gut, das sich nach rechtsstaatlichen und menschenrechtlichen Parametern bemisst, sondern eine Ware, die sich nach den Kriterien von Angebot und Nachfrage bestimmt. Im Irak berechnete Halliburton über einen Vertrag mit der bis vor kurzem zu Halliburton gehörenden Firma Kellogg, Brown \& Root (KBR) der US Army mehrfach überhöhte Benzinkosten. Und erst im März 2008 wurde bekannt, dass KBR, der größte Vertragspartner der US-Regierung im Irak, mehr als 2 I.000 Mitarbeiter über Briefkastenfirmen auf den Cayman Islands beschäftigt hat und so dem Staat hunderte Millionen Dollar Sozialabgaben und Krankenversicherung vorenthalten hat. ${ }^{19}$

Anstatt der Kostenersparnis dient das Outsourcing im Kontext der neuen Militärdoktrin primär dazu, mehrere Kriege gleichzeitig führen zu können und Militäreingriffe der öffentlichen Kontrolle zu entziehen. Gegenüber der Öffentlichkeit bietet der Einsatz von PMCs große Vorteile. Regierungen müssen sich bei unangenehmen oder illegalen Verwicklungen oder wenn es zu Toten oder Verletzten bei den Einsätzen kommt nicht verantworten, da es sich nicht um Angehörige der Streitkräfte handelt. So werden die Opferzahlen auf der eigenen Seite künstlich »niedrig « gehalten, denn weder die militärischen Auseinanderset-

I7 Eduardo Tamayo G,: Mercenarios con »impunidad de facto«, Revista Pueblos vom i 3.I I.2007.

I 8 Zitiert in: Prensa Latina vom 21.10.2007.

I9 Spiegel online vom 7.3.2008. 
zungen, in die PMC und PSC verwickelt sind, noch ihre Angestellten, die im Irak ums Leben kommen, tauchen in den einschlägigen Statistiken des US-Militärs auf. Dabei übernehmen die PMC auch direkte Kampfaufträge: Als irakische Milizen am 4. April 2004 das US-Hauptquartier in Nadschaf angriffen, dauerte das Abwehrgefecht vier Stunden, die Verteidiger schossen mit Maschinengewehren und $40 \mathrm{~mm}-$ Granaten - doch in den Berichten des US-Militärs findet sich kein Hinweis auf die Ereignisse. Der Grund: Es waren überhaupt keine Militärs an dem Gefecht beteiligt; Mitarbeiter von Blackwater hatten den Angriff abgewehrt. $^{20}$

Regierungen und damit auch das Militär sind dem Parlament gegenüber rechenschaftspflichtig, Privatfirmen nicht. Sie sind es nur gegenüber ihrem Auftraggeber. So lassen sich durch die Nutzung von PMC auch versteckte Auslandseinsätze durchführen. Da nur Aufträge mit einem Volumen von mehr als so Millionen Dollar dem US-Senat vorgelegt werden müssen, überschreiten Aufträge an PMC so gut wie nie diese Summe, so können sie jeder demokratischen Kontrolle entzogen werden. Das Pentagon verfügt mit dem Einsatz von $\mathrm{PMC}$ in zahlreichen Regionen (wie etwa Kolumbien) auch über eine direkte Kontrolle und einen direkten Zugriff auf das Kampfgebiet, während es gleichzeitig Fragen oder Kritik zurückweist, da es sich um Privatunternehmen handelt. Die Unternehmen selbst wiederum unterliegen einer vertraglich gegenüber ihrem Auftraggeber zugesicherten Schweigepflicht.

Ein weiterer Vorteil liegt in der eingangs bereits beschriebenen organisierten Straflosigkeit. Da sie nicht Militärs sind, können Angehörige von PMC und PSC nicht vor die Militärjustiz gestellt werden. Zusätzlich koppeln die meisten PMCs ihre Einsätze an einen Vertrag mit den Institutionen des Landes, in dem der Einsatz vorgenommen wird, der ihnen Immunität zusichert. Das bedeutet, dass schwere Verbrechen ungestraft bleiben. Auch im Irak ist bisher kein einziger Angestellter einer PMC juristisch belangt worden. ${ }^{21}$ Die 2005 von der UN-Menschenrechtskommission eingesetzte »Arbeitsgruppe über die Nutzung von Söldnern « stellt fest, dass die Angehörigen der PMC und PSC, obwohl schwer bewaffnet, weder Zivilisten noch Kombattanten sind. Sie repräsentierten vielmehr eine neue Form des Söldnertums, sozusagen »irreguläre Kombattanten«. Die Immunität, die verschiedene Mächte diesen Söldnern zugestehen, verwandelt sich de facto in eine Straflosigkeit. Die UN-Arbeitsgruppe warnt davor, dass die Staaten, die solche Unternehmen unter Vertrag nehmen, möglicherweise auch für die Menschenrechtsverbrechen verantwortlich sind, die das Personal dieser Firmen verübt. Angesichts der Schwierigkeiten von Staaten, in Kriegssituationen PMC und PSC zu kontrollieren, geht die Arbeitsgruppe davon aus, dass ein bedeutender Teil der Verantwortung den Staaten zufällt, von denen aus die Militär- und Sicherheitsdienstleistungen exportiert werden. Die Arbeitsgruppe fordert zudem von den Exportstaaten, den PMC und PSC und ihrem Personal keine Immunität zu verleihen. ${ }^{22}$

20 Washington Post vom 6.4.2004.

2 I United Nations, Report of the Working Group on the use of mercenaries as a means of violating human rights and impeding the exercise of the right of peoples to self-determination, I 3.9.2006, I 5 .

22 Zur Arbeit der Arbeitsgruppe siehe umfassend (Mai 2008): http://www2.ohchr.org/english/issues/mercenaries/index.htm. 
Später als viele andere Armeen arbeitet mittlerweile auch die Bundeswehr seit Ende 2007 daran, Teile der Logistik an Privatfirmen auszulagern. Diese sollen das gesamte Material der Bundeswehr lagern und die Kasernen sowie deutsche Auslandseinsätze damit beliefern (ausgenommen seien nur Munition und Sanitätsmaterial). Das sind allein im Inland 500.000 Tonnen Fracht jährlich. Der zu vergebende Auftrag, der über zehn Jahre laufen und einen Umfang von vier bis fünf Milliarden Euro haben soll, wird der größte Auftrag aller Zeiten in der deutschen Transportbranche sein. Scheinbar werden immer noch verschiedene Angebote, so z.B. der Express- und Logistiktochter der Deutschen Post DHL, des zur Deutschen Bahn gehörenden Transportunternehmens Schenker, der Dienstleistungsfirma Arvato aus der Bertelsmann-Gruppe sowie eines Konsortiums aus der Bremer Firma Hellmann Logistics, dem Flugzeug- und Rüstungskonzern EADS sowie der Beraterfirma Accenture geprüft. ${ }^{23}$ EADS gehört ohnehin schon mit CDC einem Konsortium, das der Finanzaufsicht der Deutschen Bank unterliegt und an das die Bundeswehr die Neugestaltung der Kommunikationssysteme und Datenbanken der Bundeswehr (Herkules) vergeben hat.

DHL hingegen hat bereits Erfahrungen im Irak. Zwar ist Deutschland offiziell nicht am Irakkrieg beteiligt, doch DHL bietet seit 2004 Dienstleistungen für die US Army im Irak an. Ende 2004 führte das Unternehmen bereits täglich vierzehn Flüge, mit jeweils 250-300 Tonnen Ladekapazität, in den Irak durch. Die Anzahl der DHL-Mitarbeiter im Irak stieg rapide von sieben auf I 38 an. Zunächst bestand die zentrale Aufgabe von DHL in der Auslieferung der Post für die US-Soldaten, dann übernahm DHL auch den Transport verschiedenster Güter, die vom US-Militär und unter Vertrag stehenden Unternehmen gebraucht werden. Die meisten der I 8 »ausländischen Experten « im irakischen DHL-Team haben einen militärischen Hintergrund. Zusätzlich wurde eine Gruppe ehemaliger britischer Soldaten engagiert, die, von einem »Sicherheitsmanager « koordiniert, auf das Geschäft der Posttochter aufpassen. ${ }^{24}$

Doch in Deutschland werden auch Mitarbeiter für PMC und PSC im Ausland angeworben. Das geschieht zumeist halb verdeckt, denn gesetzlich ist es verboten, Söldner für Kampfeinsätze in fremden Ländern zu rekrutieren. Es ist allerdings nicht für deutsche Staatsbürger verboten, in anderen Ländern militärisch aktiv zu werden. Wie auch in anderen Ländern sind es auch aus Deutschland vornehmlich Ex-Mitglieder von Spezialkräften von Bundeswehr und Polizei, die von PMC und PSC rekrutiert werden. So etwa aus dem Kommando Spezialkräfte (KSK), Spezialisten aus der Bundeswehr, wie Kampfschwimmer oder Scharfschützen, Ex-Angehörige von Polizei-Sondereinsatzkommandos (SEK) und GSG 9. In Deutschland erfolgt das Anwerben über Mittelsmänner oder über PSC, wie Praetoria aus Bielefeld: ein Sicherheitsunternehmen, das Schutz in Krisenregionen anbietet und nach eigenen Angaben »strategischer Sicherheitspartner« des irakischen Aufbauprogramms ist. 